\title{
Irisin Attenuates Neuroinflammation and Prevents the Memory and Cognitive Deterioration in Streptozotocin-Induced Diabetic Mice
}

\author{
Kexin Wang, ${ }^{1}$ Feng Song $\mathbb{D}^{2}{ }^{2}$ Kai Xu, ${ }^{1,3}$ Zhi Liu, ${ }^{1}$ Shuhong Han, ${ }^{4}$ Fangna Li, ${ }^{3,5}$ and Yu Sun ${ }^{5}$ \\ ${ }^{1}$ Department of General Surgery, Qilu Hospital of Shandong University, Jinan, Shandong, China \\ ${ }^{2}$ Department of Orthopedics, Qingdao Municipal Hospital, Qingdao, Shandong, China \\ ${ }^{3}$ Cheeloo College of Medicine, Shandong University, Jinan, Shandong, China \\ ${ }^{4}$ Department of Medicine, Division of Rheumatology \& Clinical Immunology, University of Florida, Gainesville, FL, USA \\ ${ }^{5}$ Department of Endocrinology, Qilu Hospital of Shandong University, Jinan, Shandong 250012, China
}

Correspondence should be addressed to Yu Sun; sunyujinan@gmail.com

Received 1 February 2019; Revised 30 March 2019; Accepted 13 May 2019; Published 9 June 2019

Guest Editor: Sun-Young Hwang

Copyright (C) 2019 Kexin Wang et al. This is an open access article distributed under the Creative Commons Attribution License, which permits unrestricted use, distribution, and reproduction in any medium, provided the original work is properly cited.

\begin{abstract}
Diabetes mellitus (DM) patients experience memory and cognitive deficits. The mechanisms underlying this dysfunction in the brain of DM patients are not fully understood, and therefore, no optimized therapeutic strategy has been established so far. The aim of the present study was to assess whether irisin was able to improve memory and cognitive performance in a streptozotocin-induced diabetic mouse model. A diabetic mouse model was established and behavioral tests were performed. We also set up primary cultures for mechanism studies. Western blots and EMSA were used for molecular studies. Significant impairment of cognition and memory was observed in these DM mice, which could be effectively prevented by irisin cotreatment. We also found upregulated levels of GFAP protein, reduced synaptic protein expression, and increased levels of interleukin-1 $\beta$ (IL-1 $\beta$ ) and interleukin-6 (IL-6) in the brains; however, irisin significantly attenuated these cellular responses. Meanwhile, our results demonstrated that irisin inhibited the activation of P38, STAT3, and NFkB proteins of DM mice. Furthermore, our results suggested that irisin might regulate the function of P38, STAT3, and NFkB in hippocampal tissues of DM mice. Collectively, irisin inhibited neuroinflammation in STZ-induced DM mice by inhibiting cytokine release and improving their cognitive function. Our findings revealed the mechanism of irisin's anti-inflammatory effect in the CNS.
\end{abstract}

\section{Introduction}

Diabetes is a chronic metabolic disorder and is becoming a global public health problem. It results in a significant increase of morbidity and mortality, placing heavy economic burdens on families and health care systems [1]. There is strong evidence that DM increases the risk of cognitive and memory deterioration [2]. Both diabetes and cognitive and memory impairment occur more commonly in elderly people. In DM, one of the prominent pathological changes is hyperglycemia that results from increased glucose production in the liver but decreased insulin production in the pancreas [3]. Such deficits likely contribute to the memory and cognitive dysfunction in DM. Clinical studies have also revealed that many pediatric and young adult patients with DM manifested lower attention and compromised executive functions [4]. Moreover, poor glycemic control in DM accelerated the rate of cognitive decline over time, while improving metabolic control could ameliorate the decline [5]. Meanwhile, around 70\%-80\% of Alzheimer's disease (AD) patients may have diabetes or abnormal glucose metabolism [6]. The rapid deterioration of cognition in diabetic patients compared to that of nondiabetic patients has been observed in a previous report [7]. These findings especially implied that the consequences of the changes in the central nervous system (CNS) of DM, such as memory and cognition 
dysfunction, were significantly due to the long-term glucose abnormality. Therefore, it is crucial to decipher the mechanism and develop new strategies to reduce the harm.

Irisin, also known as FNDC5, is a myokine that can increase energy expenditure by accelerating the "browning" of white adipose tissue [8]. Irisin is cleaved from its precursor fibronectin type III domain-containing protein 5 and is associated with better glucose homeostasis by attenuating insulin resistance [8]. Results from some studies have suggested that irisin could be a treatment option for obesity and associated diseases such as type 2 DM [9]. More importantly, recent studies demonstrated that irisin treatment improved endothelial dysfunction in diet-induced diabetic mice [10]. Endothelial dysfunction is a possible underlying mechanism by which memory and cognition deterioration is precipitated in DM animals [11]. Therefore, irisin is a promising candidate to prevent memory and cognition deterioration in diabetic patients.

Astrocytes, the most abundant cell in the CNS, support neighboring neurons and constitute the tripartite synapses [12]. Accumulating evidences have implied that astrocytes play an important role in memory and cognitive function [12]. A recent report demonstrated that repairing damaged astrocytes could improve the cognitive impairments of STZ-diabetic mice [13]. Therefore, it is reasonable to postulate that irisin exerts beneficial effects on DM mice by acting on astrocytes.

The aim of the present study is to assess (a) whether irisin can exert beneficial effects on the neuropathological changes in DM mice, specifically for astrocyte activation, neuroinflammation, and synaptic protein loss; (b) whether the astrocyte is one of the therapeutic targets of irisin; (c) whether irisin is capable of improving memory and cognitive performance and protecting neurons against astrocytemediated neuronal damage; and (d) whether irisin can regulate the signaling pathways which trigger the neuroinflammation cascade.

\section{Materials and Methods}

2.1. Animals and Drugs. We used 8-week-old male C57BL/6J mice for this animal study. All mice were on a $12 \mathrm{~h}$ light: $12 \mathrm{~h}$ dark cycle with free access to food and water. Mice were treated according to the guidelines established by the Chinese Council on Animal Care and all procedures were approved by the Animal Care Committee of Qingdao Municipal Hospital, China and Qilu Hospital of Shandong University, China.

Mice were randomly grouped into 4 groups: control, control plus irisin $(0.5 \mathrm{mg} / \mathrm{kg} /$ day $), \mathrm{STZ}(150 \mathrm{mg} / \mathrm{kg})$, and STZ plus irisin $(0.5 \mathrm{mg} / \mathrm{kg} /$ day $)$. Both STZ and irisin were bought from Sigma-Aldrich (MO, USA). STZ was dissolved in distilled $0.1 \mathrm{mmol} / \mathrm{l}$ sodium citrate buffer $(\mathrm{pH} 4.5)$ and experimental dosages of irisin were prepared in normal saline. We administered a single dose of STZ intraperitoneally to establish a diabetic mouse model. Meanwhile, irisin was given to mice through daily intraperitoneal injection as well. Behavioral tests were performed 3 weeks later.
2.2. Y Maze Test. Spatial working memory was assessed by the $Y$ maze test as previously described [14]. The experimental apparatus consisted of 3 arms ( $35 \mathrm{~cm}$ long, $25 \mathrm{~cm}$ high, and $10 \mathrm{~cm}$ wide, labeled $\mathrm{A}, \mathrm{B}$, or $\mathrm{C}$ ) diverging at a $120^{\circ}$ from the central point. The apparatus was placed $40 \mathrm{~cm}$ above the floor in a room with dim light. We individually placed mice at the end of the start arm and allowed them to move freely through the maze during an 8-minute session. A mouse was considered to have entered an arm when all 4 paws were positioned in the arm runway. The sequence of arm entries and the total number of entries over a period of $8 \mathrm{~min}$ were recorded. The percentage of alternation was defined as the number of sequential triplets containing entries into all three arms (A-B-C and A-C-B constituted a sequential triplet, while A-C-A or A-B-A did not) during the session as a proportion of the maximum possible alternation (equivalent to the total number of arm entries minus -2$) \times 100$ [14]. Mice whose total entrance number is less than 15 times during the test were not taken into the final data.

2.3. Novel Object Recognition. Nonspatial memory was measured using the novel object recognition (NOR) test as previously described [15]. The test was conducted in a Plexiglas activity box $(40 \mathrm{~cm} \times 40 \mathrm{~cm} \times 22 \mathrm{~cm})$. Mice were given 15 min daily to explore the empty box for 3 consecutive days prior to the behavioral test. On the fourth day, mice were presented with two identical objects (A1 and A2) for a period of $10 \mathrm{~min}$. After $1 \mathrm{hr}$, mice were put back into the box and exposed to two objects for $5 \mathrm{~min}$, in which one of the familiar objects (A2) was replaced by a novel object B. Animal activity was considered object exploration if the mice's nose touched the object or if the mice were facing the object within a distance of around $2 \mathrm{~cm}$ from the object [15].

2.4. Collection of Cerebrospinal Fluid (CSF) Samples. CSF was collected from the cisterna magna, as previously reported with minor modifications [12]. After anesthesia with isoflurane, we made a sagittal incision on the inferior line of the occiput. Then, we separated the subcutaneous tissue and muscles in the surrounding area to clearly expose the meninges. We could collect about 3-4 $\mu \mathrm{l}$ of CSF in the glass tube after penetrating a capillary tube into the translucent meninges.

2.5. Western Blot Analysis. Protein samples were boiled and run on SDS-PAGE gels, followed by electrophoretically transferring onto nitrocellulose membranes. The nitrocellulose membranes were then blocked with $5 \%$ nonfat dried milk in TBST buffer. The blocked membranes were incubated with an antibody to glial fibrillary acidic protein (GFAP) (1:4000; Millipore Corp., MA, USA), synaptophysin (SYP) (1:4000, Abcam, Cambridge, United Kingdom), p38 MAPK (1:1000; New England Biolabs, Beverly, MA), phospho-p38 MAPK (1:1000; New England Biolabs, Beverly, MA), and p-Stat3 (1:1000; Cell Signaling Technology, Danvers, MA) and Stat 3 (1:1000; Cell Signaling Technology, Danvers, MA) in TBST milk overnight at $4^{\circ} \mathrm{C}$. We used $\beta$-actin as a loading control (1:5000; Santa Cruz Biotechnology Inc., CA, USA). Values used for statistical analysis were 
expressed as the ratio of the band of each protein to the band of their loading control.

2.6. Enzyme-Linked Immunosorbent Assay (ELISA). We measured the concentrations of IL- 6 and IL- $1 \beta$ in the present study with an ELISA kit (eBioscience, Thermo Fisher Scientific). Each sample was loaded in a duplicate manner with appropriate dilutions to make sure their luminescent units fell within the linear range of standard curves. The values were normalized and expressed as the ratio of each sample to their total loading protein.

2.7. Electrophoretic Mobility Shift Assay (EMSA). EMSA was carried out according to a previous report with a minor modification [16]. Nuclear proteins were extracted from mouse brains. The protein of each sample was probed with excess ${ }^{32} \mathrm{P}$-end-labeled oligonucleotides with a consensus sequence specific for NF $\kappa \mathrm{B} / \mathrm{DNA}$ (Promega Corp., Madison, WI). The mixture was then fractionated on a $6 \%$ polyacrylamide gel for around 2 hours $(180 \mathrm{~V})$. The gel was then placed on filter paper and dried. After that, the gel was exposed to a film at $270^{\circ} \mathrm{C}$ for 1.5 hours followed by autoradiography.

2.8. Statistical Analysis. Data in the present study was expressed as the mean \pm SEM. The data was then analyzed with one-way ANOVA, followed by the Newman-Keuls post hoc test. A $p$ value of less than 0.05 was considered statistically significant.

\section{Results}

3.1. Irisin Improved the Memory and Cognitive Deficiency in Diabetic Mice. We tested whether DM mice could demonstrate memory and cognition deficiency in the Y maze and NOR tests. First, short-term spatial memory was investigated with the Y maze. As shown in Figure 1, DM mice showed a significant decrease of alternation behavior compared to the control mice (Figure 1(a)), while the total travel distances were comparable between these two groups (Figure 1(b)). As shown in Figure 1(a), cotreatment with irisin could significantly improve the reduced alternation in DM mice without affecting the total travel distances (Figure 1(b)). These results in the $\mathrm{Y}$ maze suggested that short-term spatial memory and spontaneous alternation were significantly reduced in the STZ-induced DM mouse model. Next, nonspatial visual discrimination memory was measured with the NOR test. The NOR test measures the ability of mice to tell between a novel object and a familiar object. To compare the performance of mice in different groups, the time spent interacting with objects (novel or familiar) was measured and a discrimination ratio was used (the ratio of time spent exploring the novel object to the time spent exploring both objects). Mice treated with STZ exhibited a low discrimination ratio (Figure 2(a)). Cotreatment with irisin prevented the STZ-induced memory deficits, while irisin alone did not affect the memory performance of mice (Figure 2(a)). The difference in the behavioral performance was not attributed to alterations in locomotion as the total distance traveled and the total time spent inter- acting with the objects did not show a significant difference between these groups (Figure 2(b)).

3.2. Irisin Inhibited the Increase of GFAP and Prevents Synaptic Protein Loss in Diabetic Mice. Astrocyte activation is closely related with cognitive dysfunction $[12,17]$. We tested with western blot whether the astrocyte activation marker, GFAP, was regulated in these DM mice. As shown in Figure 3(a), STZ treatment induced a significant increase of GFAP protein expression, while irisin could prevent the upregulated protein level. A previous study also suggested that the loss of the presynaptic vesicle protein synaptophysin (SYP) in the hippocampus correlates with cognitive decline in human patients [18]. To understand why irisin improved the behavior of DM mice, brain tissue was processed and measured with western blot to investigate whether irisin could prevent synaptic protein loss in diabetic mice. We found that STZ caused the reduced expression of SYP in DM mice, which could be effectively attenuated by irisin cotreatment (Figure 3(b))

3.3. Irisin Attenuated the $I L-1 \beta$ and $I L-6$ Levels in Hippocampal Tissues and CSF of Diabetic Mice. Since we found that irisin could inhibit astrocyte activation in DM mice and improve cognition and memory, we postulated whether neuroinflammation was a response to the neuronal deficiency in these DM mice. Firstly, we tested the expression of IL- $1 \beta$ and IL- 6 in brain hippocampal tissues of all groups. Our results indicated that STZ caused an obvious increase in IL- $1 \beta$ and IL- 6 levels in DM, which could be prevented by irisin treatment (Figure 4). Secondly, we tested the level of these two cytokines in the CSF of these mice. We found consistent results of their expression level in CSF (Figure 5). The above results suggested that irisin might attenuate the neuroinflammation in DM mice by reducing the levels of IL- $1 \beta$ and IL- 6 in their brains.

3.4. Irisin Reduced the Activation of P38, STAT3, and NFKB Proteins in the Brain Hippocampal Tissues of Diabetic Mice. We also explored the possible underlying mechanism that was responsible for the neuropathological changes in DM mice. Phosphorylation of P38 and STAT3 is closely involved in the cytokine cascade $[19,20]$. Therefore, we tested whether the activation of P38 and STAT3 was involved in the response of irisin treatment in these DM mice. The phosphorylation status of P38 and STAT3 was investigated with western blot. We found that STZ caused the increased phosphorylated protein level of P38 in the hippocampal tissues, which could be reduced by cotreatment of irisin (Figure 6(a)). The phosphorylation of STAT3 was measured in the following western blot study. As shown in Figure 6(b), STZ alone induced the upregulated expression level of phosphorylated STAT3 but cotreatment prevented the upregulation. We measured the $\mathrm{NF} \kappa \mathrm{B}$ activation with EMSA. We found that the binding activity of $\mathrm{NF} \kappa \mathrm{B}$ to DNA was significantly increased in the brain tissue of DM mice (Figure 6(c)), indicating that $\mathrm{NF} \kappa \mathrm{B}$ was activated. Treatment with irisin effectively blocked the activation of the $\mathrm{NF} \kappa \mathrm{B} / \mathrm{DNA}$ binding activity in these mice. 


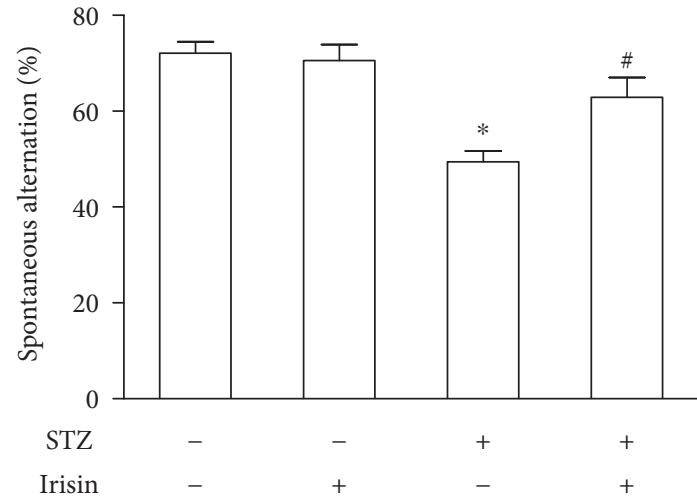

(a)

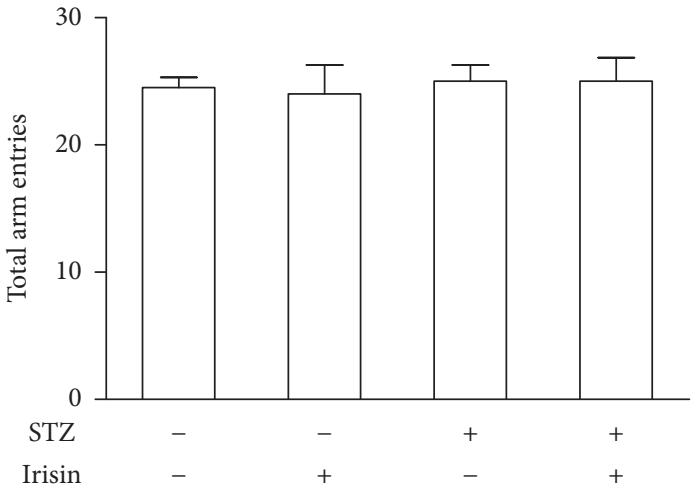

(b)

FIGURE 1: Irisin prevented STZ-induced working memory deterioration in DM mice. (a) STZ treatment caused significantly decreased spontaneous alternation of DM mice in the Y maze test. Irisin could prevent the decrease of the working memory performance of DM mice. (b) Neither STZ nor irisin could change the total arm entries of these mice. All data are expressed as means \pm SEM. $n=10$. ${ }^{*} p<0.05$ vs. STZ (-) and irisin (-); ${ }^{\#} p<0.05$ vs. STZ (+) and irisin (-).

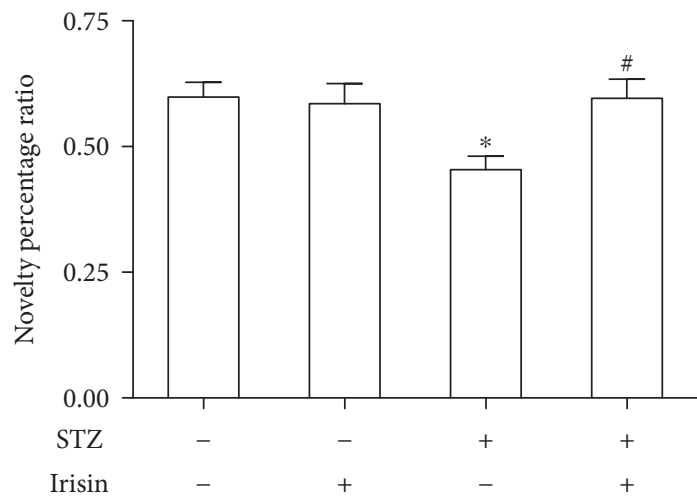

(a)

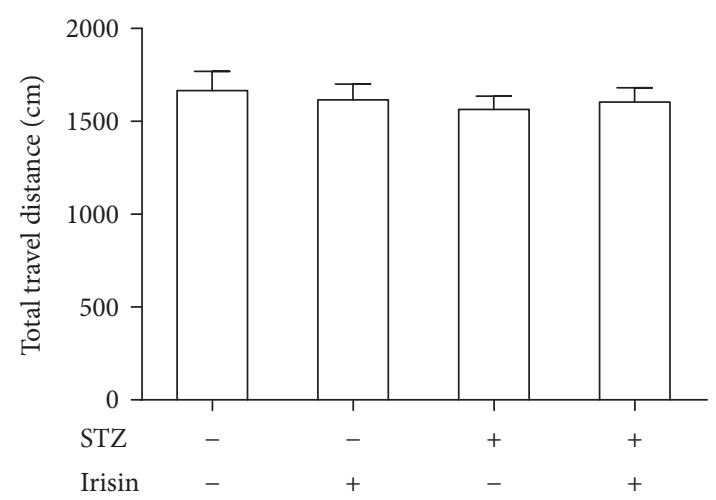

(b)

Figure 2: Irisin attenuated memory loss in STZ-induced DM mice. (a) Statistical analysis of the effect of irisin on memory deficits in DM mice. (b) There is no statistical difference of total travel distance during the test among these groups. All data are expressed as means \pm SEM. $n=10 .{ }^{*} p<0.05$ vs. STZ (-) and irisin $(-) ;{ }^{\#} p<0.05$ vs. STZ (+) and irisin (-).

\section{Discussion}

Diabetes has detrimental effects on cognitive functioning. These effects are particularly prominent for memory function [21]. However, the underlying mechanisms by which DM affects the CNS remain largely unknown. And therefore, the current therapeutic strategies are far from satisfactory due to the lack of specific medications.

In this study, we explored the possible application of irisin in the treatment of memory and cognitive dysfunction in DM. Diabetic mice were established by treating with STZ, and the effects of irisin on behavioral performances were measured with $\mathrm{Y}$ maze and NOR tests. For the first time, our data suggested that irisin could significantly improve the memory and cognitive function in DM mice (Figures 1 and 2). Although irisin is not an approved compound for the treatment of DM, a recent study has provided evidence that irisin could exert therapeutic potential in obesity and type 2 DM by stimulating the "browning" of white adipose tissue [22]. Our findings are consistent with the hypothesis that irisin might be a good candidate for the future treatment of DM since our results here also provided new evidences supporting the therapeutic benefits of irisin by improving memory and cognition in the DM mouse model.

In the CNS, especially in the hippocampus of diabetic mice, an enhanced inflammatory response with astrocyte activation has been observed, which was possibly responsible for the consequent neuronal deterioration [23]. Astrocyte activation was observed in a high-fat feeding animal model [24]. Consistently, we found a significantly increased expression of the astrocyte activation marker protein, GFAP, along with a significantly decreased level of the presynaptic protein, SYP (Figure 3). These results suggested astrocyte activation and synaptic dysfunction in these DM mice. The assumption was further partially supported by our ELISA data with the upregulated expression of two cytokines, IL- $1 \beta$ and IL-6, in brain hippocampal tissues and CSF. Interestingly, the above changes in DM mice could be effectively prevented by cotreatment with irisin. 

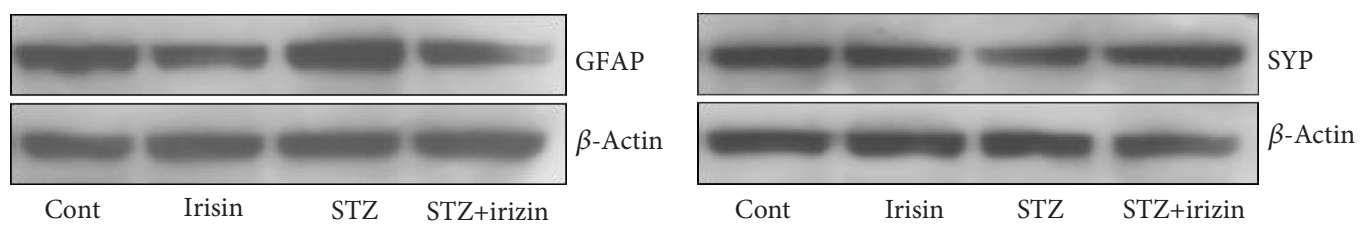

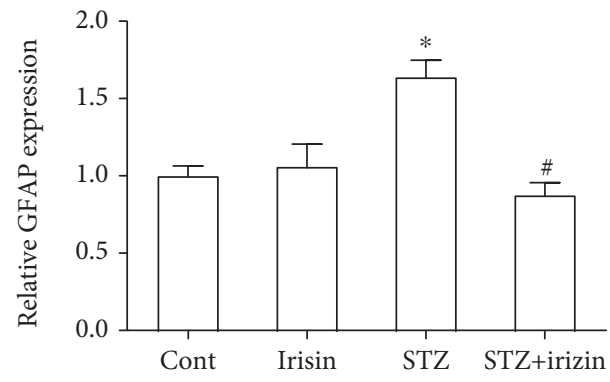

(a)

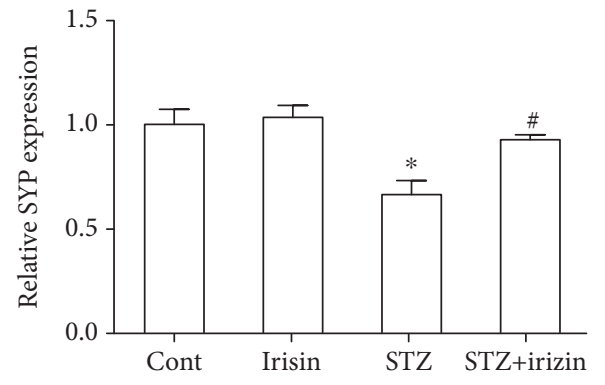

(b)

FIGURE 3: Irisin attenuates the abnormal expression level of GFAP and SYP in DM mouse exposed to STZ. (a) Representative blot picture showing the expression of the GFAP protein in DM mice and the statistical bar graph showing the results. (b) Representative blot picture showing the expression level of SYP in DM mice and the statistical bar graph showing the results. All data are expressed as means \pm SEM. $n=5 .{ }^{*} p<0.05$ vs. Cont; ${ }^{\#} p<0.05$ vs. STZ.

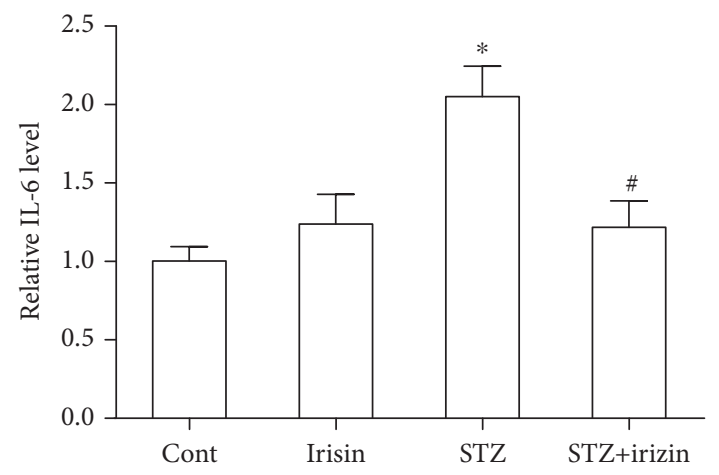

(a)

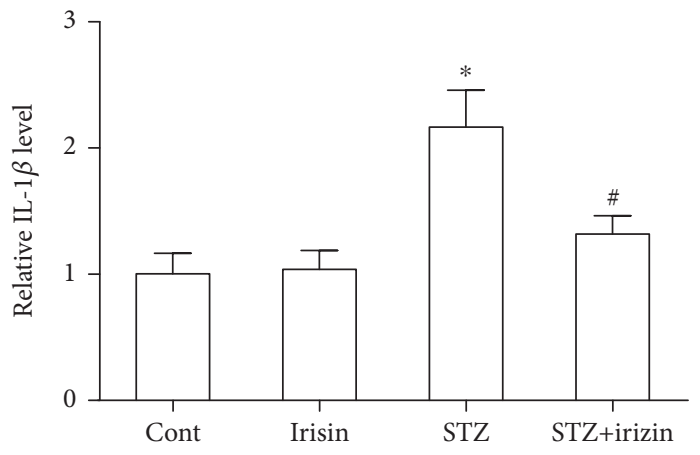

(b)

FIGURE 4: Irisin ameliorated the increased level of IL-6 and IL-1 $\beta$ in the hippocampal tissues of DM mice exposed to STZ. (a) Statistical bar graph showing irisin reduced the STZ-induced increased expression level of IL-6 in hippocampal tissues of DM mice. (b) Statistical bar graph showing irisin reduced the STZ-induced increased expression level of IL-1 $\beta$ in hippocampal tissues of DM mice. All data are expressed as means \pm SEM. $n=6 .{ }^{*} p<0.05$ vs. Cont; ${ }^{\#} p<0.05$ vs. STZ.

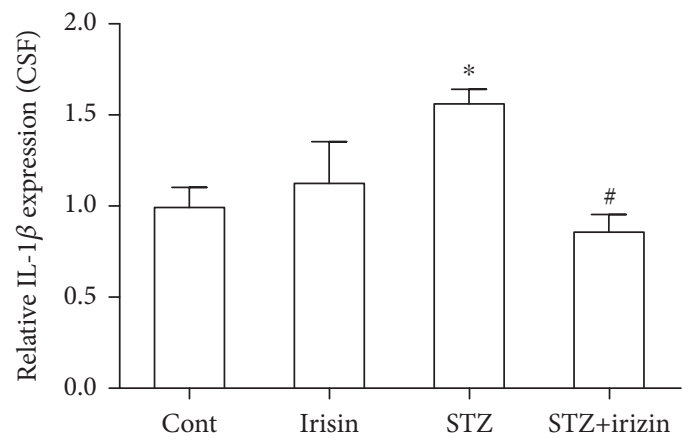

(a)

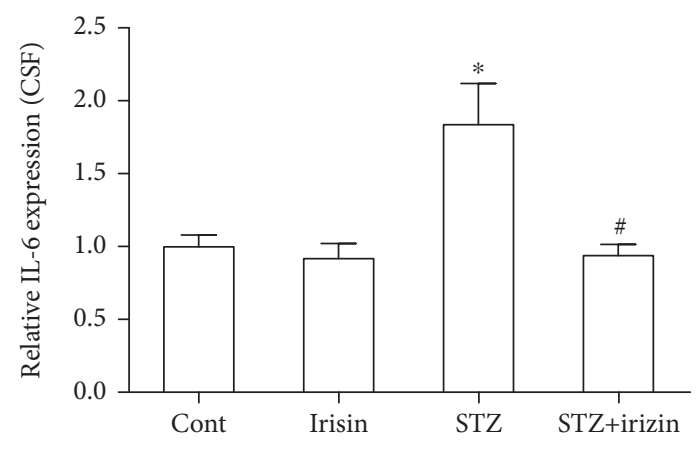

(b)

FIGURE 5: Irisin reduced the level of IL- 6 and IL- $1 \beta$ in the CSF of DM mice exposed to STZ. (a) STZ increased the level of IL- $1 \beta$ in the CSF of DM mice, but irisin treatment could reduce the upregulation of IL-1 $\beta$. (b) STZ increased the level of IL-6 in the CSF of DM mice, and irisin treatment could reduce the upregulation of IL-6. All data are expressed as means \pm SEM. $n=6 .{ }^{*} p<0.05$ vs. Cont; ${ }^{\#} p<0.05$ vs. STZ. 

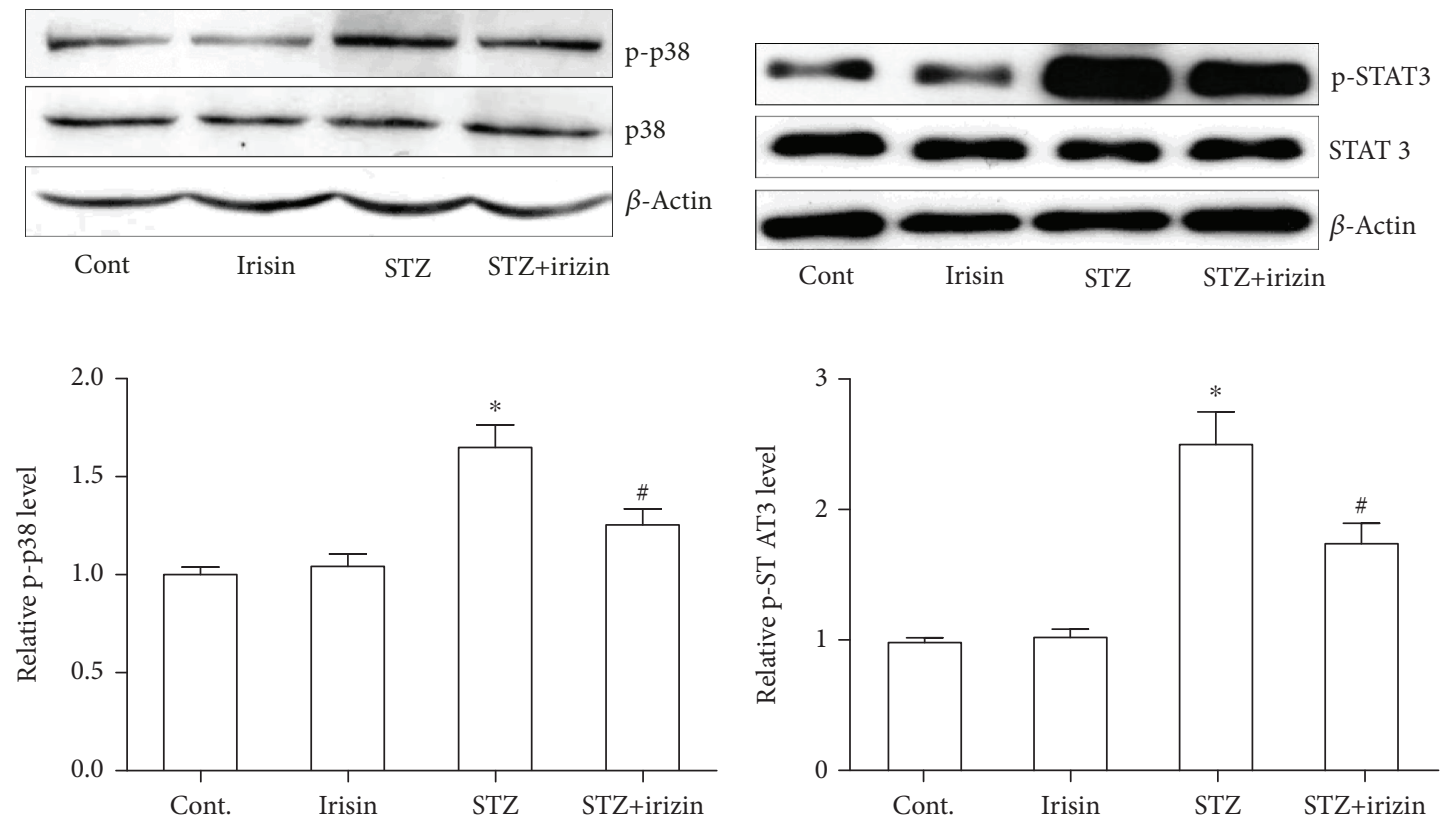

(a)

(b)

Probe Cont Irisin STZ STZ+irizin

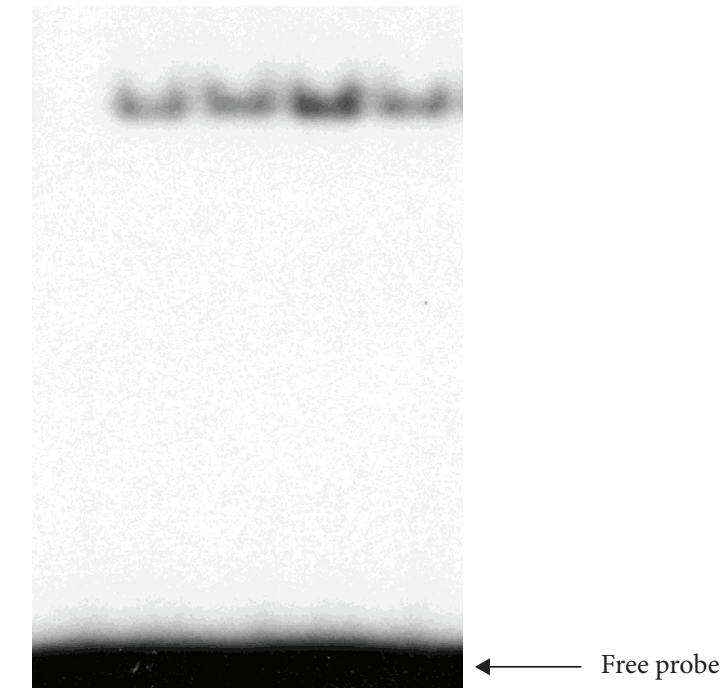

(c)

FIGURE 6: Irisin reduced the activation of p38, STAT3, and NF $\kappa$ B proteins in the brain hippocampal tissues of DM mice. (a) Representative western blot photograph and statistical results of p-p38 and total p38 in hippocampal tissues of all groups. (b) Representative western blot photograph and statistical results of p-STAT3 and total STAT3 in hippocampal tissues of all groups. (c) NF $\kappa$ B/DNA binding activity was determined by EMSA. All data are expressed as means \pm SEM. $n=5 .{ }^{*} p<0.05$ vs. Cont; ${ }^{*} p<0.05$ vs. STZ.

Previous studies have well established the role of p38 and STAT3 in neuroinflammation $[25,26]$. In the present study, notable changes of the phosphorylation level of p38 and STAT3 were shown in a western blot study (Figure 6). STAT3 is one of key factors involved in many cytokine cascades, including IL-6, IL-10, and TNF $\alpha$ [27]. And a recent study disclosed that the JAK/STAT and STAT3 pathway played a key role in the induction of NMDA-receptordependent long-term depression in the hippocampus [28]. Moreover, the STAT3 in astrocytes is an essential step by which astrocytes play a proinflammatory response in the CNS [29]. Our findings here further highlighted the importance of STAT3 in memory and cognitive function, especially in the pathological setting, such as in elderly patients with DM.

To the best of our knowledge, the present study provides the first evidence that irisin prevents memory and cognitive deficits via regulating JAK/STAT and STAT3 and consequent inflammatory injury in the brains of DM mice. We anticipate that our findings here will provide the foundation 
for future clinical trials to determine whether irisin may prevent or treat memory and cognitive dysfunction in DM patients while attenuating glucose abnormality.

\section{Data Availability}

The data used to support the findings of this study are available from the corresponding author upon request.

\section{Conflicts of Interest}

All authors declare that there are no conflicts of interest in this study.

\section{Acknowledgments}

This study was financially supported by the National Natural Science Foundation of China (Grant Nos. 81471020 and 81400770), the Shandong Medical and Health Technology Development Project (Grant No. 2014WS0148), Shandong University Basic Scientific Research Funding (Qilu Hospital Clinical Research Project) (Grant No. 2014QLKY21), and Qilu Hospital of Shandong University Scientific Research Funding (Grant No. 2015QLMS32).

\section{References}

[1] K. M. V. Narayan, E. W. Gregg, A. Fagot-Campagna, M. M. Engelgau, and F. Vinicor, "Diabetes - a common, growing, serious, costly, and potentially preventable public health problem," Diabetes Research and Clinical Practice, vol. 50, Supplement 2, pp. S77-S84, 2000.

[2] E. Saedi, M. R. Gheini, F. Faiz, and M. A. Arami, "Diabetes mellitus and cognitive impairments," World Journal of Diabetes, vol. 7, no. 17, pp. 412-422, 2016.

[3] D. A. Butterfield, F. Di Domenico, and E. Barone, "Elevated risk of type 2 diabetes for development of Alzheimer disease: a key role for oxidative stress in brain," Biochimica et Biophysica Acta (BBA) - Molecular Basis of Disease, vol. 1842, no. 9, pp. 1693-1706, 2014.

[4] C. M. Ryan, E. van Duinkerken, and C. Rosano, "Neurocognitive consequences of diabetes," American Psychologist, vol. 71, no. 7, pp. 563-576, 2016.

[5] O. Ojo and J. Brooke, "Evaluating the association between diabetes, cognitive decline and dementia," International Journal of Environmental Research and Public Health, vol. 12, no. 7, pp. 8281-8294, 2015.

[6] J. Janson, T. Laedtke, J. E. Parisi, P. O'Brien, R. C. Petersen, and P. C. Butler, "Increased risk of type 2 diabetes in Alzheimer disease," Diabetes, vol. 53, no. 2, pp. 474-481, 2004.

[7] R. Ravona-Springer, X. Luo, J. Schmeidler et al., "Diabetes is associated with increased rate of cognitive decline in questionably demented elderly," Dementia and Geriatric Cognitive Disorders, vol. 29, no. 1, pp. 68-74, 2010.

[8] N. Perakakis, G. A. Triantafyllou, J. M. Fernández-Real et al., "Physiology and role of irisin in glucose homeostasis," Nature Reviews Endocrinology, vol. 13, no. 6, pp. 324-337, 2017.

[9] T. Hofmann, U. Elbelt, and A. Stengel, "Irisin as a musclederived hormone stimulating thermogenesis - A critical update," Peptides, vol. 54, pp. 89-100, 2014.
[10] N. Hou, G. Du, F. Han, J. Zhang, X. Jiao, and X. Sun, "Irisin Regulates Heme Oxygenase-1/Adiponectin Axis in Perivascular Adipose Tissue and Improves Endothelial Dysfunction in Diet-Induced Obese Mice," Cellular Physiology and Biochemistry, vol. 42, no. 2, pp. 603-614, 2017.

[11] B. Sharma and N. Singh, "Pitavastatin and 4-Hydroxy-3Methoxyacetophenone (HMAP) Reduce Cognitive Dysfunction in Vascular Dementia During Experimental Diabetes," Current Neurovascular Research, vol. 7, no. 3, pp. 180-191, 2010.

[12] J. Qiao, J. Wang, H. Wang et al., "Regulation of astrocyte pathology by fluoxetine prevents the deterioration of Alzheimer phenotypes in an APP/PS1 mouse model," Glia, vol. 64, no. 2, pp. 240-254, 2016.

[13] M. Nakano, K. Nagaishi, N. Konari et al., "Bone marrowderived mesenchymal stem cells improve diabetes-induced cognitive impairment by exosome transfer into damaged neurons and astrocytes," Scientific Reports, vol. 6, no. 1, 2016.

[14] J. Wang, S. Zhu, H. Wang et al., “Astrocyte-dependent protective effect of quetiapine on GABAergic neuron is associated with the prevention of anxiety-like behaviors in aging mice after long-term treatment," Journal of Neurochemistry, vol. 130, no. 6, pp. 780-789, 2014.

[15] J. Wang, Y. Zhang, H. Xu et al., "Fluoxetine improves behavioral performance by suppressing the production of soluble $\beta$-amyloid in APP/PS1 mice," Current Alzheimer Research, vol. 11, no. 7, pp. 672-680, 2014.

[16] H. Y. Hsiao, O. T. Mak, C. S. Yang, Y. P. Liu, K. M. Fang, and S. F. Tzeng, "TNF- $\alpha /$ IFN- $\gamma$-induced iNOS expression increased by prostaglandin E2 in rat primary astrocytes via EP2-evoked cAMP/PKA and intracellular calcium signaling," Glia, vol. 55, no. 2, pp. 214-223, 2007.

[17] G. Dallérac and N. Rouach, "Astrocytes as new targets to improve cognitive functions," Progress in Neurobiology, vol. 144 , pp. $48-67,2016$.

[18] C.-I. Sze, J. C. Troncoso, C. Kawas, P. Moution, D. L. Price, and L. J. Martin, "Loss of the Presynaptic Vesicle Protein Synaptophysin in Hippocampus Correlates with Cognitive Decline in Alzheimer Disease," Journal of Neuropathology and Experimental Neurology, vol. 56, no. 8, pp. 933-944, 1997.

[19] Y. Wang, A. H. H. van Boxel-Dezaire, H. Cheon, J. Yang, and G. R. Stark, "STAT3 activation in response to IL-6 is prolonged by the binding of IL-6 receptor to EGF receptor," Proceedings of the National Academy of Sciences, vol. 110, no. 42, pp. 16975-16980, 2013.

[20] D. C. Underwood, R. R. Osborn, C. J. Kotzer et al., “SB 239063, a potent p38 MAP kinase inhibitor, reduces inflammatory cytokine production, airways eosinophil infiltration, and persistence," Journal of Pharmacology and Experimental Therapeutics, vol. 293, no. 1, pp. 281-288, 2000.

[21] M. Kumari, E. Brunner, and R. Fuhrer, "Minireview: mechanisms by which the metabolic syndrome and diabetes impair memory," The Journals of Gerontology Series A, vol. 55, no. 5, pp. B228-B232, 2000.

[22] P. Boström, J. Wu, M. P. Jedrychowski et al., "A PGC1- $\alpha$ dependent myokine that drives brown-fat-like development of white fat and thermogenesis," Nature, vol. 481, no. 7382, pp. 463-468, 2012.

[23] A. Nagayach, N. Patro, and I. Patro, "Astrocytic and microglial response in experimentally induced diabetic rat brain," Metabolic Brain Disease, vol. 29, no. 3, pp. 747-761, 2014. 
[24] L. B. Buckman, M. M. Thompson, R. N. Lippert, T. S. Blackwell, F. E. Yull, and K. L. J. Ellacott, "Evidence for a novel functional role of astrocytes in the acute homeostatic response to high-fat diet intake in mice," Molecular Metabolism, vol. 4, no. 1, pp. 58-63, 2015.

[25] J. P. O'Callaghan, K. A. Kelly, R. L. VanGilder, M. V. Sofroniew, and D. B. Miller, "Early activation of STAT3 regulates reactive astrogliosis induced by diverse forms of neurotoxicity," PLOS ONE, vol. 9, no. 7, article e102003, 2014.

[26] X. Guo, C. Harada, K. Namekata et al., "Regulation of the severity of neuroinflammation and demyelination by TLRASK1-p38 pathway," EMBO Molecular Medicine, vol. 2, no. 12 , pp. 504-515, 2010.

[27] C. F. Lai, J. Ripperger, K. K. Morella et al., "Receptors for interleukin (IL)-10 and IL-6-type cytokines use similar signaling mechanisms for inducing transcription through IL-6 response elements," Journal of Biological Chemistry, vol. 271, no. 24, pp. 13968-13975, 1996.

[28] C. S. Nicolas, S. Peineau, M. Amici et al., "The Jak/STAT pathway is involved in synaptic plasticity," Neuron, vol. 73, no. 2, pp. 374-390, 2012.

[29] Y. Lu, M. He, Y. Zhang et al., "Differential pro-inflammatory responses of astrocytes and microglia involve STAT3 activation in response to $1800 \mathrm{MHz}$ radiofrequency fields," PLOS ONE, vol. 9, no. 10, article e108318, 2014. 


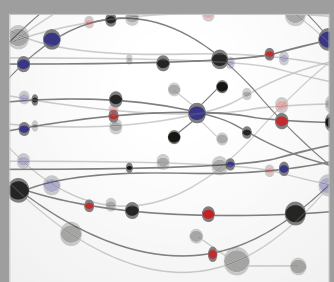

The Scientific World Journal
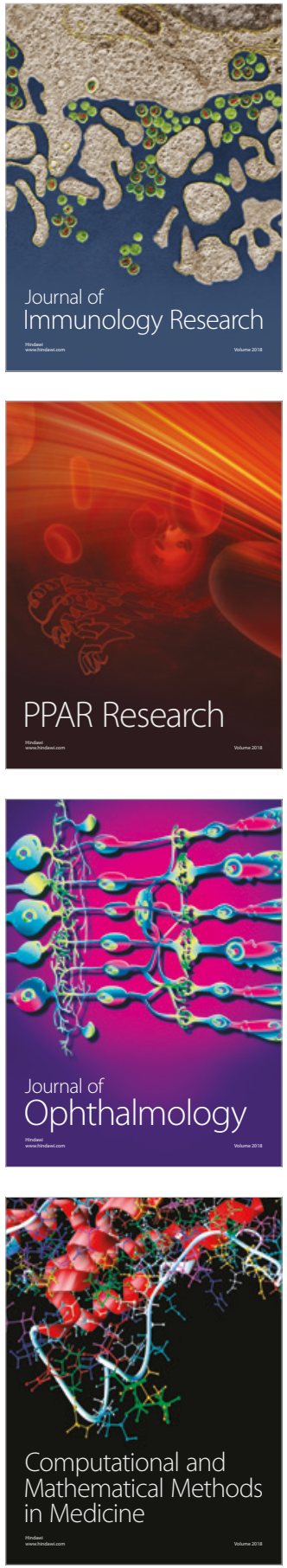

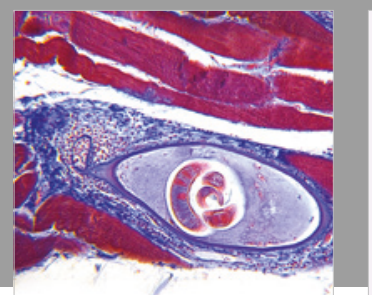

Gastroenterology Research and Practice

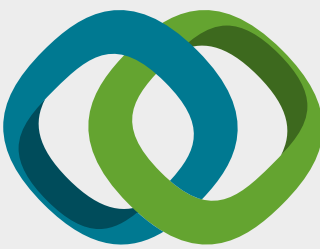

\section{Hindawi}

Submit your manuscripts at

www.hindawi.com
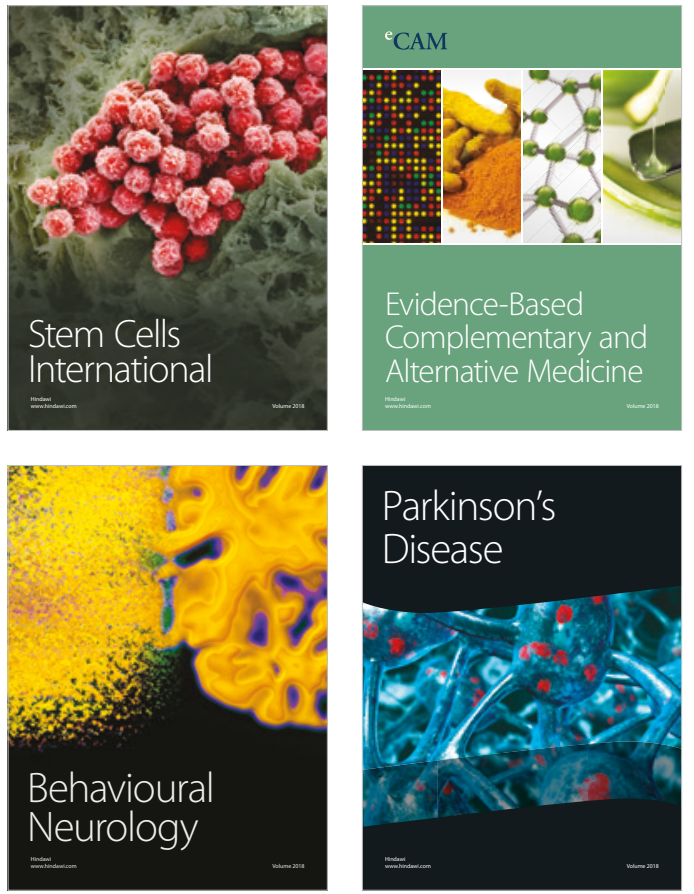

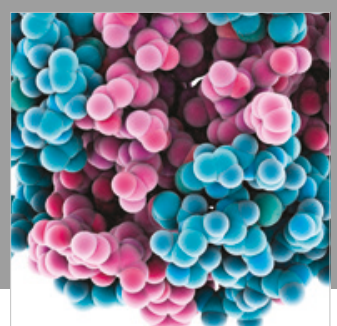

ournal of

Diabetes Research

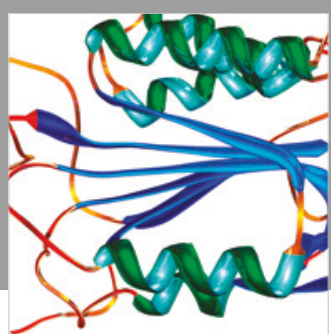

Disease Markers
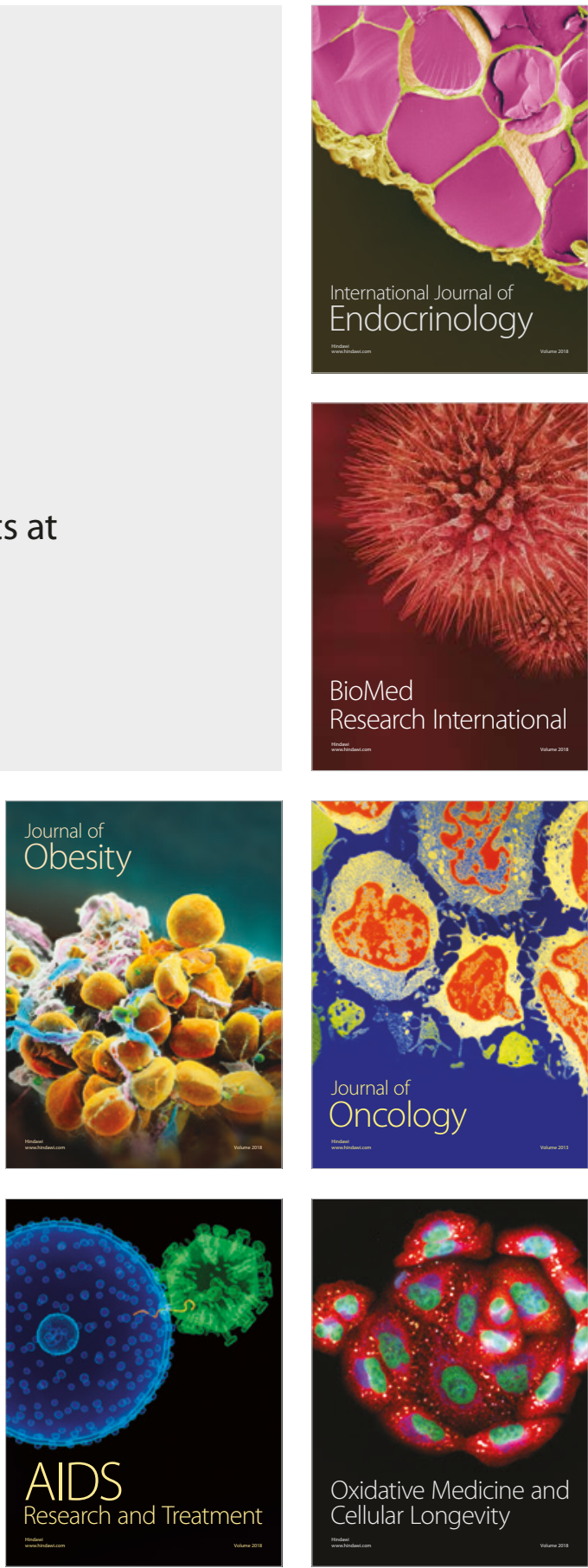